\title{
Natural Products (Apigenin, Curcumin and Emodin) Cure Lung Cancer
}

\author{
Bashir Ahmad ${ }^{2,3 *}$, Pengyu Su${ }^{1}$, Shah Zeb Khan ${ }^{6}$, Fazal Wahid ${ }^{4}$, Hayat Ullah ${ }^{4}$, Manzoor Ahmad \\ Badshah Hussainn ${ }^{5}$ Jamshed Khan ${ }^{5}$, Syed Rafiq Hussain Shah ${ }^{2}$ and Shahab Uddin ${ }^{4}$ \\ ${ }^{1}$ Institute of Cancer Stem Cell \& The Second Affiliated Hospital, Dalian Medical University, China \\ ${ }^{2}$ College of Basic Medical Science, Dalian Medical University, P. R. China
}

${ }^{3}$ Department of Zoology, Shaheed Benazir Bhuto University Sheringal, District Dir (Upper) Khyber Pakhtunkhwa, Pakistan

${ }^{4}$ Department of Botany, University of Malakand Chakdara, Pakistan

${ }^{5}$ Department of Botany, Hazara University, Pakistan

${ }^{6}$ Department of Botany, Shaheed Benazir Bhuto University Sheringal, District Dir (Upper) Khyber Pakhtunkhwa, Pakistan

${ }^{7}$ Department of Chemistry, University of Malakand Chakdara, Pakistan

*Corresponding author: Bashir Ahmad, College of Basic Medical Science, Dalian Medical University, P. R. China.

To Cite This Article: Bashir Ahmad. Natural Products (Apigenin, Curcumin and Emodin) Cure Lung Cancer. 2020 - 9(2). AJBSR.MS.ID.001375. DOI: 10.34297/AJBSR.2020.09.001375.

Received: 阱April 28, 2020; Published: 眥 June 18, 2020

\begin{abstract}
Lung cancer is the most common malignancy worldwide with over 1.8 million new cases diagnosed each year. As overall survival is only $15 \%$ for five years, therefore the appropriate therapy is compulsory for its treatment. A number of therapies are in practice for its treatment, but natural products (NPs) are considered safe and effective. Among these NPs, Apigenin, Curcumin and Emodin are reported for the treatment of lung cancer; therefore we summarized the studies of these NPs against lung cancer. This review will provide an outline for further research on these compounds against lung cancer.
\end{abstract}

Keywords: Lung cancer, Natural product, Apigenin, Curcumin, Emodin

\section{Introduction}

Lung cancer is the most common malignancy worldwide with over 1.8 million new cases diagnosed each year [1]. Lung cancer incidence is the second highest while the leading cause of mortality in the United States. Approximately 85\% lung cancers are NSCLC Lung cancers. Nearly $80 \%$ of patients have regional or distal spread of disease at the time of diagnosis, accounting for poor overall survival [2]. Lung cancer screening is important and can shift the diagnosis to earlier and surgically resect able stage with significantly improved outcomes and cure but patients diagnosed with NSCLC are usually older and often have multiple age-smoking related comorbidities, poor functional status borderline function, all factors that affect prognosis and health management [3]. Overall survival is poor and only around $15 \%$ of patients are alive
5 years after their initial diagnosis [1]. Lung cancer is among the malignancies with poor prognosis. In 2015, lung cancer was the fifth leading cause of mortality in United States, with the World Health Organization (WHO) reporting 1.7 million deaths worldwide [4].

A number of therapies are in practice for the treatment of cancer, natural products (plants derived compounds) are considered less toxic and more effective. [5] Medicinal plants are the vital sources for NPs $[6,7]$ and it cure cancer through modulation of multiple pathways, including oxidative stress, intrinsic, extrinsic apoptosis pathway, cell cycle, inflammation, NF-kB, MEK-ERK etc. 6 Among these NPs, Apigenin, Curcumin and Emodin are reported for the treatment of lung cancer; therefore we summarized the studies of these NPs against lung cancer. 


\section{Apigenin}

Apigenin (AP) inhibits the proliferation and induced apoptosis in NCI-H460 cells time and dose dependently through inhibition of BCL-2 and upregulation of BAX and Caspase-3 [8]. In sphere forming NCI-H446 cells AP inhibit the self-renewal capacity which may have link with the down-regulation of UPAR expression [9]. AP inhibit the A549 cells proliferation and inhibit tumour growth through down-regulation of HIF-1 and VEGF [10]. AP alone or in combination with leptin inhibit the A549 cells proliferation and induces apoptosis through increase in ROS generation [11]. AP induces apoptosis in A549 cell though reactive oxygen species (ROS) generation, Mitochondrial membrane depolarization, $\mathrm{Bcl} 2$ down-regulation and Bax up-regulation, Cytochrome c (cyt c) decrease in mitochondrial fraction due to release to cytosol as result up-regulate the caspase 3,9 and PARP which lead to DNA fragmentation [12]. AP inhibit the proliferation of A549 and H460 NSCLC calls through downregulation of Axl and Xiap and upregulation of p21 and cyclin-dependent kinase inhibitor [13]. Another study reveal that the AP decrease the A549 cells viability and induces morphological changes time and dose-dependently through release of cyt-c, AIF and Endo G lead to activation of caspase-3,9 [14]. In H460 cells, AP induces apoptosis through ROS generation and $\mathrm{Ca} 2 \mathrm{t}$, MMP dissipation, down regulation of Bid, Bcl2, procaspase-8, upregulation of Bax, AIF, cyt-c, caspase-3, GRP78 and GADD153 [15]. AP sensitize H1299 and A549 cells to TRIALinduced apoptosis through upregulation of death receptor 4 (DR4) and death receptor 5 (DR5) in p53 dependent manner which lead to the upregulation of Bax and Bad, downregulate the Bclxl and Bcl-2.

Furthermore AP inhibit NF-kB, AKT and ERK activation. In xenograft model, AP and TRIAL in combination completely suppress the tumour growth compared to its alone treatment [16]. We show that AP significantly decreases GLUT1 expression in mice. Furthermore, AP induces growth retardation and apoptosis through metabolic and oxidative stress caused by suppression of glucose utilization in lung cancer cells. The underlying mechanisms were defined that the anticancer effects of AP were reversed by ectopic GLUT1 overexpression and galactose supplementation, through activation of pentose phosphate pathway-mediated NADPH generation. Importantly, we showed that severe metabolic stress using a glutaminase inhibitor, compound 968, was involved in the mechanism of sensitization by AP. Taken together, the combination of AP with inhibitors of glutamine metabolism may provide a promising therapeutic strategy for cancer treatment [17]. AP tocopherol derivative-containing D-alpha-tocopheryl polyethylene glycol 1000 succinate (TPGS) liposomes and tyroservicate in combination exhibit superior cyto-toxicity, suppress invasion of A549 cells, induced G2 arrest. Furthermore, the combinatory effect inhibits the tumour growth in A549 cells bearing mice [18]. In A549 cells, AP exert anti-proliferative, anti- migration and anti-vision trough PI3K/Akt signaling pathway and inhibition of its down-stream signaling genes expression of matrix metalloproteinases-9, glycogen synthase kinase-3 $\beta$, and HEF1 [19]. Taken together the AP is a potential natural compound for the lung cancer chemoprevention.

\section{Curcumin}

Curcumin (Cum) induces apoptosis in human lung cancer cells through various mechanisms including synergistic induction of cell death and apoptosis with AP and also by blocking cell cycle progression at growth/mitotic (G2/M) phase of A549 cells [20]. showed that by binding to purified microtubules Cum can depolymerize interphase microtubules and inhibit reassembly of cold depolymerized microtubules [20]. Another study demonstrated that the Cum induces apoptosis in A549 cells through downregulation of Microribonucleic acid (miRNA) and caspase-10 which is identified as a target of miRNA-186 [21-23] showed that Cum down-regulate the miRNA level in A549 cells especially miR186 and led the cells to apoptosis [23,24]. Examined the in vitro effects of Cum on human lung cancer cell line apoptosis. They showed that Cum increases the growth arrest and DNA damage (GADD) 45 and 153 in a p53-independent manner and also inhibits the growth of PC-9 cells and induces G(1)/S arrest of the cell cycle followed by strong induction of apoptosis. They further showed that expression of cyclin dependent kinase inhibitor genes p21 and p27 is induced by Cum, while the expression of numerous other genes, including Bcl-2, cyclin D1, CDK2, CDK4, and CDK6, is inhibited by Cum. Upregulation of GADD45 and 153by Cum is one of the prime mechanisms of its anticancer activity [24]. Taken together all this information, Cum is potential therapeutic NP for the treatment of lung cancer.

\section{Emodin}

In TCM, constituents of rhubarb (Rheum palmatum) have found a wide range of therapeutic applications including antitumor [25]. Major compounds of therapeutic importance in rhubarb are derivatives of anthraquinone, including emodin [26]. Many reports suggest that emodin efficiently suppresses multiple cell signaling pathways and also inhibits cell proliferation, invasion, metastasis, and angiogenesis [27]. Emodin is the most important therapeutic agent for the treatment of NSCLC. In NSCLC, emodin increase the cisplatin induced toxicity through inhibition of induced ERK1/2 activation and ERCC1 protein induction via instability of ERCC1 protein [28]. In NCL-H446 small cell lung cancer cells, emodin suppressed the viability, increase the apoptosis and induced changes in cell cycle through down-regulation of 8 genes and upregulation of 10 gene after 12 hours and 12 gene up-regulate and 24 were down regulated after $24 \mathrm{~h}$. These genes were involved in signal transduction, metabolism, cytoskeleton organization, transport, cell adhesion, transcription regulation, immune response, cell 
adhesion, RNA processing and cell cycle control [29]. Emodin exert a suppressive effect on NSCLC proliferation in concentration dependent manner through down regulation of Rad51 and ERCC1 [30]. Emodin induces growth inhibition and apoptosis in A549 cells through extrinsic pathway in which emodin upregulate the FASL while downregulate the C-myc and lead to DNA fragmentation [31]. In H1299 and A549 cells the emodin decrease cells viability and induces apoptosis through tribbles homolog 3 (TRIB3) induced ER stresses [32]. These studies show that the emodin has very potential therapeutic effect against lung cancer.

\section{Conclusion}

Lung cancer is the most common malignancy around the world. NPs cure the lung cancer through different mechanisms. Among these NPs, AP, Cum and Emodin has very potential effect for the treatment of lung cancer.

\section{Conflict of Interest}

The authors declare no conflict of interest.

\section{Consent for publication}

All authors agree to be published.

\section{Reference}

1. Ferlay J, Soerjomataram I, Ervik M, Dikshit R, Eser S, et al. (2014) GLOBOCAN 2012 v1. 0, Cancer Incidence and Mortality Worldwide: IARC Cancer Base No. 11. International Agency for Research on Cancer, Lyon, France.

2. (2015) Situ BCI: Cancer Facts.

3. Siegel RL, Miller KD, Jemal A (2015) Cancer statistics. CA Cancer J Clin 65: 5-29.

4. (2014) The top 10 causes of death: World Health Organization. World Health Organization.

5. Pengyu S AB, Lijuan Z (2018) Natural $\beta$-Element: Advances in Targeting Cancer Through Different Molecular Pathways. North American Journal of Acedamic Research 1: 27.

6. Ahmad B, Khan S, Liu Y, Xue M, Nabi G, et al. (2020) Molecular Mechanisms of Anticancer Activities of Puerarin. Cancer management and research 12: 79-90.

7. Jalal S, Ahmad B, Zhang T, Guo L, Huang L, et al. (2020) SANTAMARINE: Mechanistic Studies on Multiple Diseases. Chem Biol Drug Des 95(4): 427-434.

8. Pan X, Yang Z, Yang Z, Zhou S, Zhang H, et al. [Effect of apigenin on proliferation and apoptosis of human lung cancer NCI-H460 cells]. Nan fang yi ke da xue xue bao 33(8): 1137-1140.

9. Shu L, Yuan Q, Cui Y, Sun S, Chen A, et al. (2016) [Effects of apigenin on self-renewal and uPAR expression in NCI-H446 cell line]. Zhong nan da xue xue bao Yi xue ban 41:1124-1127.

10. Liu LZ, Fang J, Zhou Q Hu X, Shi X, et al. (2005) Apigenin inhibits expression of vascular endothelial growth factor and angiogenesis in human lung cancer cells: implication of chemoprevention of lung cancer. Mol Pharmacol 68: 635-643.

11. Bruno A, Siena L, Gerbino S, Ferraro M, Chanez P, et al. (2011) Apigenin affects leptin/leptin receptor pathway and induces cell apoptosis in lung adenocarcinoma cell line. European journal of cancer 47(13): 20422051.
12. Das S, Das J, Samadder A, Boujedaini N, Khuda-Bukhsh AR, et al. (2012) Apigenin-induced apoptosis in A375 and A549 cells through selective action and dysfunction of mitochondria. Exp Biol Med (Maywood) 237(12): 1433-1448

13. Kim KC, Choi EH, Lee C (2014) Axl receptor tyrosine kinase is a novel target of apigenin for the inhibition of cell proliferation. Int J Mol Med 34(2): 592-598.

14. Lu HF, Chie YJ, Yang MS, Lee CS, Fu JJ, et al. (2010) Chung JG: Apigenin induces caspase-dependent apoptosis in human lung cancer A549 cells through Bax- and Bcl-2-triggered mitochondrial pathway. Int J Oncol 36: 1477-1484.

15. Lu HF, Chie YJ, Yang MS, Lu KW, Fu JJ, et al. (2011) Apigenin induces apoptosis in human lung cancer $\mathrm{H} 460$ cells through caspase- and mitochondria-dependent pathways. Human \& experimental toxicology 30(8): 1053-1061.

16. Chen M, Wang X, Zha D, Cai F, Zhang W, et al. (2016) Apigenin potentiates TRAIL therapy of non-small cell lung cancer via upregulating DR4/DR5 expression in a p53-dependent manner Sci Rep 6: 35468.

17. Lee YM, Lee G, Oh TI, Kim BM, Shim DW, et al. (2016) Inhibition of glutamine utilization sensitizes lung cancer cells to apigenin-induced apoptosis resulting from metabolic and oxidative stress. International journal of oncology 48(1): 399-408.

18. Jin X, Yang Q, Zhang Y (2017) Synergistic apoptotic effects of apigenin TPGS liposomes and tyroservatide: implications for effective treatment of lung cancer. Int J Nanomedicine 12: 5109-5118.

19. Zhou Z, Tang M, Liu Y, Zhang Z, Lu R, et al. (2017) Apigenin inhibits cell proliferation, migration, and invasion by targeting Akt in the A549 human lung cancer cell line. Anticancer Drugs 28(4): 446-456.

20. Choudhury D, Ganguli A, Dastidar DG, Acharya BR, Das A, et al. (2013) Apigenin shows synergistic anticancer activity with curcumin by binding at different sites of tubulin. Biochimie 95(6): 1297-1309.

21. Zhang J, Du Y, Wu C, Ren X, Ti X, et al. (201) Curcumin promotes apoptosis in human lung adenocarcinoma cells through miR-186* signaling pathway. Oncology reports 24(5): 1217-1223.

22. Zhang J, Zhang T, Ti X, Shi J, Wu C, et al. (2010) Curcumin promotes apoptosis in A549/DDP multidrug-resistant human lung adenocarcinoma cells through an miRNA signaling pathway. Biochem Biophys Res Commun 399(1): 1-6.

23. Tang N, Zhang J, Du Y (2010) [Curcumin promoted the apoptosis of cisplain-resistant human lung carcinoma cells A549/DDP through down-regulating miR-186*]. Zhongguo fei ai za zhi 13(4): 301-306.

24. Saha A, Kuzuhara T, Echigo N, Fujii A, Suganuma M, et al. (2010) Apoptosis of human lung cancer cells by curcumin mediated through up-regulation of "growth arrest and DNA damage inducible genes 45 and 153". Biological \& pharmaceutical bulletin 33(8): 1291-1299.

25. Shang XY, Yuan ZB (2002) [Determination of six effective components in Rheum by cyclodextrin modified micellar electrokinetic chromatography]. Yao xue xue Bao 37: 798-801.

26. Shang X, Yuan Z (2003) Determination of hydroxyanthraquinoids in Rhubarb by cyclodextrin-modified micellar electrokinetic chromatography using a mixed micellar system of sodium dodecyl sulfate and sodium cholate. Journal of pharmaceutical and biomedical analysis 31(1): 75-81.

27. Tan W, Lu J, Huang M, Li Y, Chen M, et al. (2011) Anti-cancer natural products isolated from chinese medicinal herbs. Chin Med 6(1): 27.

28. Ko JC, Su YJ, Lin ST, Jhan JY, Ciou SC, et al. (2010) Emodin enhances cisplatin-induced cytotoxicity via down-regulation of ERCC1 and inactivation of ERK1/2. Lung cancer 69(2): 155-164.

29. Fu ZY, Han JX, Huang HY (2007) Effects of emodin on gene expression profile in small cell lung cancer NCI-H446 cells. Chinese medical journal 120(19): 1710-1715 
30. He L, Bi JJ, Guo Q, Yu Y, Ye XF (2012) Effects of emodin extracted from Chinese herbs on proliferation of non-small cell lung cancer and underlying mechanisms. Asian Pac J Cancer Prev APJCP 13(14): 1505-1510.

31. Li WY, Ng YF, Zhang H, Guo ZD, Guo DJ, et al. (2014) Emodin elicits cytotoxicity in human lung adenocarcinoma A549 cells through inducing apoptosis. Inflammopharmacology 22(2): 127-134.
32. Su J, Yan Y, Qu J, Xue X, Liu Z, et al. (2017) Emodin induces apoptosis of lung cancer cells through ER stress and the TRIB3/NF-kappaB pathway. Oncology reports 37(3): 1565-1572. 